\title{
A New Method for Producing Graded Index PMMA Waveguides
}

Victor White, Reza Ghodssi, Gregory Fish, Cheryl Herdey, Henley Liu, Denice D. Denton, and Leon McCaughan, Senior Member, IEEE

\begin{abstract}
We report a new method for producing graded index polymethyl methacrylate (PMMA) waveguides using optical densification by angled $x$-ray exposure. The resulting larger refractive index near the surface of the waveguide serves to better isolate the guided light from potentially absorbing or scattering substrates than in waveguides of constant refractive index. We calculate that a $65^{\circ}$ angle exposure in $6-\mu \mathrm{m}$-thick planar PMMA waveguide shifts the peak of the fundamental mode profile $\sim 0.5 \mu \mathrm{m}$ away from the substrate. Consistent with this result, we measure a reduction in the propagation loss of PMMA planar waveguides on a Ni substrate from $1.5 \mathrm{~dB} / \mathrm{cm}$ to $0.5 \mathrm{~dB} / \mathrm{cm}$ after angle $x$-ray exposure.
\end{abstract}

\section{INTRODUCTION}

$\mathbf{P}$ OLYMERIC optical waveguides offer a batch-processcompatible method for producing guided wave optical interconnects for electronic and optoelectronic integrated circuitry. Unlike silica-on silicon $\left(\mathrm{SiO}_{2} / \mathrm{Si}\right)$ waveguides, PMMA processing temperatures will not influence previously fabricated elements, nor do they exhibit the polarization dependence caused by intrinsic strain birefringence [1]. To avoid losses due to absorption by the substrate or scattering of the guided light at the film/substrate interface, it is usually necessary to place an intervening layer of lower refractive index between the guiding region and the substrate, or to otherwise grade the transverse index profile (e.g., [2] and [3])

We report a simple, one-step method for producing a graded refractive index profile normal to the surface of PMMA thin films. This technique is based on the fact that exposure of PMMA to soft $x$-rays increases its refractive index (Fig. 1). This index increase is likely due to a densification of the polymer. Exposing the PMMA at an angle $(\theta)$ to the surface normal increases the effective path length of the $x$-rays, making the $\mathrm{x}$-ray dose decrease more rapidly with depth $(\sim \exp (-\alpha d / \cos \theta))$, where $\alpha(\lambda)$ is the absorption coefficient of $\mathrm{x}$-rays by PMMA and $d$ is the thickness of the PMMA film. The result is a continuously graded refractive index profile which, for large exposure angles, should be significantly larger at the upper surface than at the PMMA/substrate interface. Light will, therefore, tend to be isolated from the substrate by the lower index at the PMMA/substrate interface, reducing absorption and scatter from the substrate. $\mathrm{He}^{+}$ion implantation

Manuscript received January 26, 1995; revised March 3, 1995. This lette was supported in part by NSF Grant ECS-9215070, and by the CXrL at the Synchrotron Radiation Center of the University of Wisconsin-Madison.

The authors are with the Department of Electrical and Computer Engineering, University of Wisconsin. Madison. WI 53706 USA

IEEE Log Number 9412168 .

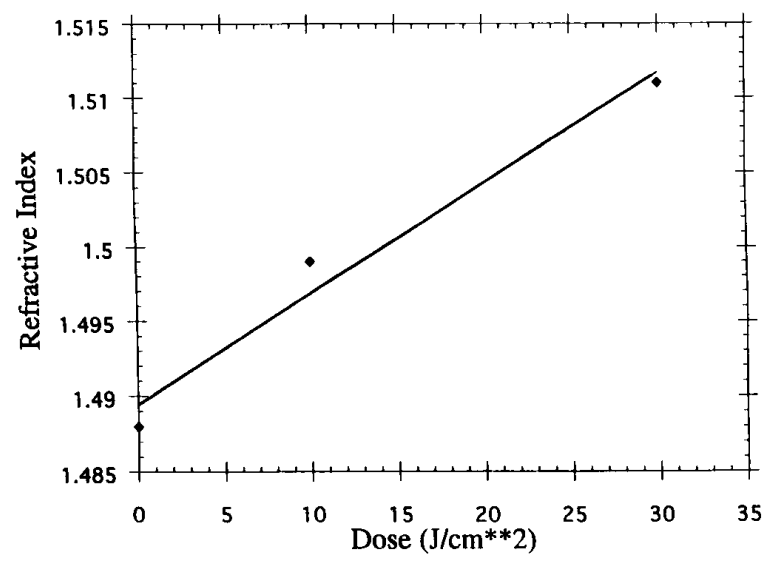

Fig. 1. Plot of the refractive index as a function of $\mathrm{x}$-ray dose in $0.2-\mu \mathrm{m}$ PMMA films as measured by ellipsometry.

at normal incidence to a PMMA film has recently been reported to have a similar effect [4], although no waveguiding properties were measured.

Refractive index changes and optical waveguide propagation losses were measured in spin-coated PMMA as a function of $\mathrm{x}$-ray exposure. The PMMA was deposited on 3-in oxidized (100) silicon wafers that were then coated with $\sim 0.5 \mu \mathrm{m}$ of electroplated nickel. The wafers were then heated to $160^{\circ} \mathrm{C}$ at $1{ }^{\circ} \mathrm{C} / \mathrm{min}$ and annealed for one hour. Film thickness was measured with a profilometer. Wafers were placed under 20 torr He pressure and exposed to a broadband $\mathrm{x}$-ray source ( $\sim 900-1300 \mathrm{eV}$ at the Alladin Storage Ring, University of Wisconsin).

Fig. 1 shows the refractive index change with exposure in thin $(0.2 \mu \mathrm{m})$ films as measured by an ellipsometer (Auto-el) at $632.8 \mathrm{~nm}$. PMMA sensitivity is seen to be about $1250 \mathrm{~J} / \mathrm{cm}^{2}$ per unity index change. Using the absorption spectrum of PMMA, $\alpha(E)$, and the intensity spectrum of the Alladin x-ray source, $I(E)$, we calculated [5] the x-ray dose (i.e., absorbed energy) as a function of the depth for a $6-\mu \mathrm{m}$ thick PMMA film irradiated with $12 \mathrm{~J} / \mathrm{cm}^{2}$ at $65^{\circ}$ to the surface normal (typical exposure time $\sim 10 \mathrm{~min}$ ). The resulting refractive index profile was calculated from the data in Fig. 1 and is shown in Fig. 2. Also shown in the figure is the calculated field intensity profile of the fundamental mode (at $\lambda=632 \mathrm{~nm}$ ) of the exposed (i.e., the exponential index profile) film. Not unexpectedly, the peak intensity of the fundamental mode in the exponentially graded waveguide is moved $\sim 0.5 \mu \mathrm{m}$ away from the substrate, 


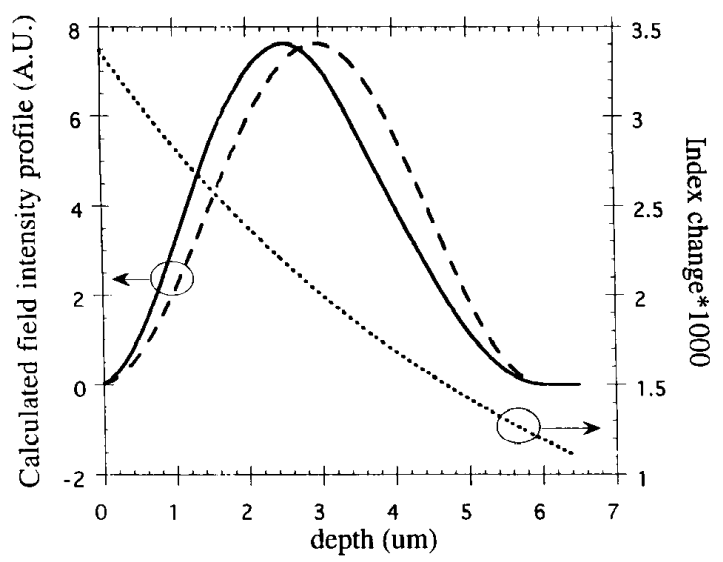

Fig. 2. Plots of the refractive index profile (dotted line) and the consequen fundamental mode intensity field profiles at $632.8 \mathrm{~nm}$ for unexposed (dashed line) and angle-exposed (solid line) PMMA.

relative to the optical profile in an ungraded guide. Therefore, for the case of a PMMA waveguide on a lossy substrate, we would expect a reduction in the propagation loss after the angle $\mathrm{x}$-ray exposure.

To verify this model, HeNe light $(632.8 \mathrm{~nm})$ was prismcoupled [6] into 6- $\mu$ m-thick PMMA (multimode) films fabricated on electroplated Ni substrates. Propagation losses were determined by measuring the intensity of the light scattered normal to the waveguide as a function of position along the guide [7]. Fig. 3 is a comparison of the measured propagation losses in 6- $\mu \mathrm{m}$ PMMA planar waveguides with and without a $65^{\circ}$ angle-exposure to $x$-rays. The angle-exposed waveguides exhibit lower loss $(0.5 \mathrm{~dB} / \mathrm{cm})$ than the unexposed guides $(1.5 \mathrm{~dB} / \mathrm{cm})$. These results are consistent with the idea that the light field is better isolated from the substrate in the (x-ray-produced) graded index profile. Nevertheless, some of the reduction in loss may also arise from a change in the material properties (e.g., density) of the PMMA film produced by the $\mathrm{x}$-ray exposure. Angled $\mathrm{x}$-ray exposure provides a convenient post-process method for producing graded index

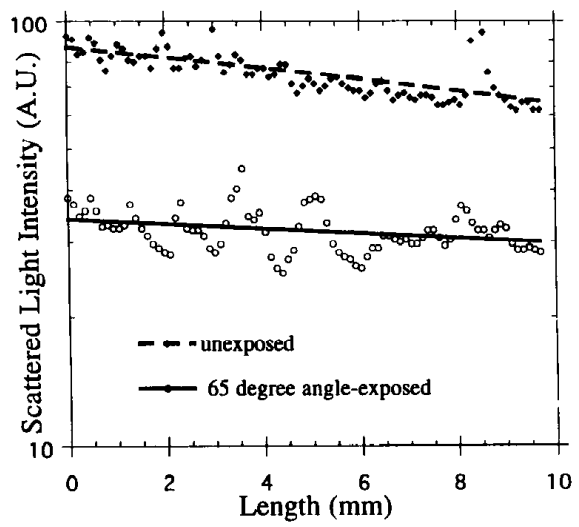

Fig. 3. Propagation loss measurements of $6 \mu \mathrm{m}$ PMMA planar waveguides without $\mathrm{x}$-ray exposure (dashed line) and after a $12 \mathrm{~J} / \mathrm{cm}^{2} \mathrm{x}$-ray exposure at $65^{\circ}$ angle of incidence (solid line).

waveguides. In addition to better isolating the light from lossy substrates, it should be possible to produce single mode waveguides, even with thick PMMA layers.

\section{REFERENCES}

[1] Y. Inoue, K. Katoh, and M. Kawachi, "Polarization sensitivity of a silica waveguide thermooptic phase shifter for planar lightwave circuits," Photon. Technol. Lett., vol. 4, pp. 36-38, 1992.

[2] R. T. Chen, "Graded index linear and curved polymer channel waveguide arrays for massively parallel optical interconnects," Appl. Phys. Lett., vol. 61, pp. 2278-2280, 1992.

[3] P. D. Townsend, G. L. Baker, N. E. Schlotter, C. F. Klausner, and S. Eternad, "Waveguiding in spun films of soluble polydiacetylenes," Appl. Phys. Lett., vol. 53, pp. 1782-1784, 1988.

[4] L. Zhang, P. D. Townsend, P. J. Chandler, and J. R. Kulisch, "Ion implanted waveguides in polymethylmethacrylate," J. Appl, Phys., vol. 66, pp. 4547-4548, 1989.

[5] F. Cerrina, F. Baszler, S. Turner and M. Khan, Microelectronic Engineering, vol. 21, pp. 103-106, 1993.

[6] R. G. Hunsperger, Integrated Optics: Theory and Technology, 2nd ed., Springer Series in Optical Sciences. New York: Springer Verlag, 1984.

[7] F. Zernike, "Fabrication and measurement of passive components," in Top. Appl. Phys., Integrated Opt. New York: Springer Verlag, 1985, vol. 7 , p. 216 